\title{
Hydro-elastic vibration analysis of functionally graded rectangular plate in contact with stationary fluid
}

\author{
Shahrouz Yousefzadeh ${ }^{a}$, Ashkan Akbaria and Mohammad Najafi \\ aDepartment of Mechanical Engineering, Aligudarz Branch, Islamic Azad University, Aligudarz, Iran; \\ ${ }^{b}$ Department of Mechanical and Aerospace Engineering, Science and Research Branch, Islamic Azad \\ University, Tehran, Iran
}

\begin{abstract}
This study investigates the free vibration of a moderately thick rectangular plate, which is composed of functionally graded materials and floating on incompressible fluid. Material properties are assumed to be graded in the thickness direction according to a simple power law distribution in terms of the volume fraction of the constituent. The governing equations of the plate are analytically derived based on the first-order shear deformation theory with consideration of rotational inertial effects and transverse shear stresses. Applied pressure on the free surface of the plate is obtained by the velocity potential function together with Bernoulli's equation. The equation governing on the oscillatory behaviour of the fluid is obtained by solving Laplace equation with satisfying the boundary conditions. The natural frequencies and shape modes of the rectangular plate are determined by decoupling and solving the motion equations system. Then, analyses of the numerical results of free vibrations and the effects of the different parameters such as thickness to length of the plate, boundary conditions, fluid density, index of volume fraction and the height of the fluid on the frequencies are investigated. Finally, the results of this research in limit case is compared and validated with the results of other researchers and finite element model.
\end{abstract}

\section{ARTICLE HISTORY}

Received 20 November 2017

Accepted 9 July 2018

\section{KEYWORDS}

Free vibration; rectangular plate; natural frequency; functionally graded material

\section{Introduction}

The plates are the resistant structures which use extensively in industries. Evaluation of the plate dynamic characteristics is a practical engineering problem and has attracted the attention of many researches (Hejripour \& Saidi, 2012). Fluid-structure interaction problems can be found in many engineering fields, and hydro-elastic characteristics of plates coupled with fluid are important in various engineering applications such as in nuclear engineering, liquid storage tanks, reactor internal components and solar plates and offshore naval or marine structures. Thus, a good understanding of the 
dynamic interactions between an elastic plate and fluid is necessary (Hasheminejad, Khaani, \& Shakeri, 2013). It is generally well known that the natural frequencies of structures coupled with fluid are different from those in air (Kerboua, Lakis, Thomas, \& Marcouiller, 2008). During the past decades, different approximate solutions have been presented by investigators to predict the natural frequencies of structures coupled with fluid. There have been many theoretical and experimental studies devoted to vibration analysis of plates coupled with fluid. Hosseini-Hashemi et al. studied free vibration of functionally graded (FG) rectangular plate using the first-order shear deformation plate theory. They presented analytical solution for vibration of FG plate on Winkler elastic foundation (Hosseini-Hashemi, Taher, Akhavan, \& Omidi, 2010). Uğurlu et al. studied the dynamic behaviour of rectangular plates mounted on an elastic foundation and in partial contact with a quiescent fluid. They employed the mixed-type finite element formulation to present the natural frequencies and associated mode shapes of the plate (Uğurlu, Kutlu, Ergin, \& Omurtag, 2008). Allahverdizadeh et al. developed a semi-analytical approach for nonlinear free and forced axisymmetric vibration of thin circular FG plate. They solved the governing equations of plate by using assumed-time-mode and Kantrovich method for harmonic vibrations (Allahverdizadeh, Naei, \& Bahrami, 2008). The natural frequencies of annular plates coupled with a fluid on one side were theoretically obtained by Amabili et al. using the added-mass approach, whereas the coupled fluid-structure system was solved by adopting the Hankel transform (Amabili, Frosali, \& Kwak, 1996). Triverdilo et al. derived added mass and frequencies for asymmetric free vibration of coupled system including clamped circular plate coupled with incompressible bounded fluid. They used two approaches to derive the free vibration frequencies of the system (Tariverdilo, Shahmardani, Mirzapour, \& Shabani, 2013). Jeong dealt with the free vibration of two identical circular plates coupled with a bounded or unbounded fluid. $\mathrm{He}$ suggested an analytical method based on the finite Fourier-Bessel expansion and Rayleigh-Ritz method. Also, the effect of gap between the plates on the fluid-coupled natural frequencies is investigated (Jeong, 2003). Khorshidi and Bakhsheshi analysed the free natural frequencies of an FG composite rectangular plate coupled with fluid in two directions. They calculated the natural frequencies of the plate coupled with sloshing fluid modes using RayleighRitz method and based on minimising the Rayleigh quotient (Khorshidi \& Bakhsheshy, 2014). Dong investigated three-dimensional free vibrations of FG annular plates with different boundary conditions using the ChebyshevRitz method by choosing a set of duplicate Chebyshev polynomial series multiplied by the boundary function satisfying the boundary conditions (Dong, 2008). Kwak analysed the virtual mass effect due to the presence of water on the natural frequencies of circular plates. He obtained the nondimensional added virtual mass incremental factors by employing the integral 
transformation technique in conjunction with the Fourier-Bessel series approach (Kwak, 1997). More recently, Jeong et al. developed a theoretical Reyleih-Ritz-based method to estimate the coupled natural frequencies of a vertical clamped circular plate partially coupled with an ideal incompressible liquid (Jeong, Lee, \& Kim, 2009). Hosseini-Hashemi et al. analysed the free vibration analysis of a horizontal rectangular plate, either immersed in fluid or floating on its free surface. The governing equations for a moderately thick rectangular plate analytically were derived based on the Mindlin's plate theory, whereas the velocity potential function and Bernoulli's equation were employed to obtain the fluid pressure applied on the free surface of the plate. In this work, an exact closed-form characteristic equation was used for the plate subjected to a combination of six different boundary conditions (Hosseini-Hashemi, Karimi, \& Rokni, 2012).

A new class of materials knew as functionally graded material (FGM) has been introduced in which the material properties vary continuously in one or more directions according to the specific profile. The development of analytic solutions to identify the dynamic characteristic of FG plates coupled with fluid is a challenge task. The above review clearly indicates that while there exists a notable body of literature on the free vibrational characteristics of fluid-coupled homogeneous plates, there seem to be no rigorous theoretical or numerical solutions describing free vibrations of fluid-coupled FG plates. This paper presents a theory to calculate the natural frequencies of a circular FG plate partially contacting bounded fluid, using Reyleih-Ritz method. In the developed model, the first-order shear deformation theory is used to obtain the kinetic and strain energies of the plate. Wet dynamic transverse displacement of the plate is approximated by a set of admissible trial functions which is required to satisfy the boundary conditions. The equations governing the oscillatory behaviour of fluid are obtained by solving Laplace equation with satisfying boundary conditions. The natural frequencies and mode shapes of the coupled fluid modes are calculated by using Chebyshev-Ritz method based on minimising the Rayleigh quotient. The proposed analytical method is verified by comparing the results obtained by finite element analysis. Finally, the influence of boundary conditions, plate and tank dimensions, fluid density and depth on frequencies is discussed in details.

\section{Formulation}

\subsection{Formulation for rectangular plate}

Figure 1 shows a flat and moderately thick FG rectangular plate partially coupled with incompressible fluid. $\mathrm{a}, \mathrm{b}$ and $\mathrm{h}$ represent the length, width and thickness of the rectangular plate, respectively, and $h_{1}$ refers to depth 


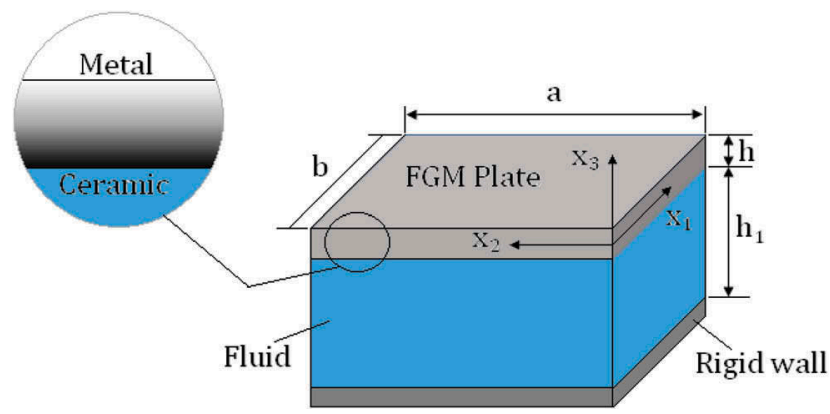

Figure 1. Rectangular plate coupled with cubic fluid with coordinate convection.

of the fluid. The Cartesian coordinate system $\left(x_{1}, x_{2}, x_{3}\right)$ is considered to extract mathematical formulations when $x_{1}$ and $x_{2}$ axes are located in the undeformed mid-plane of the plate.

The displacement along the $x_{1}$ and $x_{2}$ axes is denoted by $U_{1}$ and $U_{2}$, respectively, and the displacement in the direction perpendicular to the undeformed mid-plane $x_{3}$ is denoted by $U_{3}$. According to Mindlin's plate theory in Cartesian coordinate $\left(x_{1}, x_{2}, x_{3}\right)$, the displacement components are assumed to be given by (Hashemi, Karimi, \& Taher, 2010):

$$
\begin{aligned}
& \boldsymbol{U}_{1}=-\boldsymbol{x}_{3} \psi_{1}\left(\boldsymbol{x}_{1}, \boldsymbol{x}_{2}, \boldsymbol{t}\right) \\
& \boldsymbol{U}_{2}=-\boldsymbol{x}_{3} \psi_{2}\left(\boldsymbol{x}_{1}, \boldsymbol{x}_{2}, \boldsymbol{t}\right) \\
& \boldsymbol{U}_{3}=\boldsymbol{\psi}_{3}\left(\boldsymbol{x}_{1}, \boldsymbol{x}_{2}, \boldsymbol{t}\right)
\end{aligned}
$$

where $\psi_{1}$ and $\psi_{2}$ are the rotational displacements about the $x_{1}$ and $x_{2}$ axes at the midsurface, respectively, $\psi_{3}$ is the transverse displacement and $t$ is the time variable. Under the assumption of small deformation and linear strain-displacement relations, the strain components of the FG rectangular plate can be expressed as:

$$
\begin{aligned}
\varepsilon_{11} & =\frac{\partial U_{1}}{\partial x_{1}}=-x_{3} \psi_{1,1} \\
\varepsilon_{22} & =\frac{\partial U_{2}}{\partial x_{2}}=-x_{3} \psi_{2,2} \\
\varepsilon_{33} & =\frac{\partial U_{3}}{\partial x_{3}}=0 \\
\varepsilon_{12} & =\frac{1}{2}\left(\frac{\partial U_{1}}{\partial x_{2}}+\frac{\partial U_{2}}{\partial x_{1}}\right)=-\frac{1}{2}\left(\psi_{1,2}+\psi_{2,1}\right) x_{3} \\
\varepsilon_{13} & =\frac{1}{2}\left(\frac{\partial U_{1}}{\partial x_{3}}+\frac{\partial U_{3}}{\partial x_{1}}\right)=-\frac{1}{2}\left(\psi_{1}-\psi_{3,1}\right) \\
\varepsilon_{23} & =\frac{1}{2}\left(\frac{\partial U_{3}}{\partial x_{2}}+\frac{\partial U_{2}}{\partial x_{3}}\right)=-\frac{1}{2}\left(\psi_{2}-\psi_{3,2}\right)
\end{aligned}
$$


Considering the plane stress state for FG plate, the stresses are defined as follows:

$$
\begin{aligned}
\sigma_{11} & =\frac{E\left(x_{3}\right)}{1-v^{2}}\left(\varepsilon_{11}+v \varepsilon_{22}\right) \\
\sigma_{22} & =\frac{E\left(x_{3}\right)}{1-v^{2}}\left(\varepsilon_{22}+v \varepsilon_{11}\right) \\
\sigma_{12} & =\frac{E\left(x_{3}\right)}{2(1+v)}\left(2 \varepsilon_{12}\right) \\
\sigma_{23} & =\frac{E\left(x_{3}\right)}{2(1+v)}\left(2 \varepsilon_{23}\right) \\
\sigma_{13} & =\frac{E\left(x_{3}\right)}{2(1+v)}\left(2 \varepsilon_{13}\right)
\end{aligned}
$$

where $v$ is Poisson's ratio and is considered to be constant and $E\left(x_{3}\right)$ is Young's modulus and is assumed to vary continuously throughout the thickness of the FG plate in $x_{3}$-direction. According to the power law function, $E\left(x_{3}\right)$ is written as below (Jomehzadeh, Saidi, \& Atashipour, 2009):

$$
E\left(x_{3}\right)=E_{m}+\left(E_{c}-E_{m}\right)\left(\frac{1}{2}-\frac{x_{3}}{h}\right)^{p}
$$

in which $p$ is the power law index that takes values greater than or equal to zero. The subscripts $m$ and $c$ refer to the metal and ceramic, respectively. Note that the variation of both continuous of ceramic and metal is linear when $p=1$. Moreover, for the value of $p=0$, a fully ceramic plate is intended. Based on the strain-displacement relations given in Equation (2) and assuming a stress distribution in accordance with Hook's law, the resultant bending moments, twisting moments and the transverse shear forces, all per unit length in terms of $\psi_{1}, \psi_{2}$ and $\psi_{3}$, are obtained by integrating the stresses and moment of the stresses through the thickness of the plate. These are given by:

$$
\begin{aligned}
& M_{11}=\int_{-h / 2}^{h / 2} \sigma_{11} x_{3} d x_{3}=-D\left(\psi_{1,1}+v \psi_{2,2}\right) \\
& M_{22}=\int_{-h / 2}^{h / 2} \sigma_{22} x_{3} d x_{3}=-D\left(\psi_{2,2}+v \psi_{1,1}\right) \\
& M_{12}=\int_{-h / 2}^{h / 2} \sigma_{12} x_{3} d x_{3}=-\frac{D(1-v)}{2}\left(\psi_{1,2}+\psi_{2,1}\right) \\
& Q_{1}=\int_{-h / 2}^{h / 2} \sigma_{13} d x_{3}=-\frac{A(1-v) \kappa^{2}}{2}\left(\psi_{1}-\psi_{3,1}\right) \\
& Q_{2}=\int_{-h / 2}^{h / 2} \sigma_{23} d x_{3}=-\frac{A(1-v) \kappa^{2}}{2}\left(\psi_{2}-\psi_{3,2}\right)
\end{aligned}
$$

in which $\kappa^{2}$ is the transverse shear correction coefficient, applied to the transverse shear forces due to the fact that the transverse shear strains $\left(\varepsilon_{13}\right.$ 
and $\varepsilon_{23}$ ) have a nearly parabolic dependency to the thickness coordinate and it is assumed to be $\pi^{2} / 12$. Also, the parameters $A, B$ and $D$ are defined in the following form:

$$
(A, B, D)=\int_{-\mathrm{h}_{/ 2}}^{\mathrm{h} / 2} \frac{E\left(x_{3}\right)}{1-v^{2}}\left(1, x_{3}, x_{3}^{2}\right) \mathrm{d} x_{3}
$$

According to Navier-Stokes equation, the governing equations of the rectangular plate motion in Cartesian coordinate system can be given:

$$
\begin{aligned}
& \frac{\partial}{\partial x_{1}} \sigma_{11}+\frac{\partial}{\partial x_{2}} \sigma_{12}+\frac{\partial}{\partial x_{3}} \sigma_{13}=\rho\left(x_{3}\right) \frac{\partial^{2} U_{1}}{\partial t^{2}} \\
& \frac{\partial}{\partial x_{1}} \sigma_{21}+\frac{\partial}{\partial x_{2}} \sigma_{22}+\frac{\partial}{\partial x_{3}} \sigma_{23}=\rho\left(x_{3}\right) \frac{\partial^{2} U_{2}}{\partial t^{2}} \\
& \frac{\partial}{\partial x_{1}} \sigma_{31}+\frac{\partial}{\partial x_{2}} \sigma_{32}+\frac{\partial}{\partial x_{3}} \sigma_{33}=\rho\left(x_{3}\right) \frac{\partial^{2} U_{3}}{\partial t^{2}}
\end{aligned}
$$

Multiplying the first and second equations to $x_{3} d x_{3}$ and third equation to $d x_{3}$, integrating through the thickness of the plate and using Equation (5), the following equations are obtained:

$$
\begin{aligned}
& M_{11,1}+M_{12,2}-Q_{1}=-I_{3} \psi_{1} \\
& M_{12,1}+M_{22,2}-Q_{2}=-I_{3} \psi_{2} \\
& Q_{1,1}+Q_{2,2}-P=I_{1} \psi_{3}
\end{aligned}
$$

In the above equations, a dot above each parameter denotes partial differentiation with respect to time variable $t$ and $I_{i}(i=1,2,3)$ are inertia terms which are defined as:

$$
I_{1}, I_{2}, I_{3}=\int_{-h_{/ 2}}^{h_{/ 2}} \rho\left(x_{3}\right)\left(1, x_{3}, x_{3}^{2}\right) \mathrm{d} x_{3}
$$

where $\rho\left(x_{3}\right)$ is the density of FG plate which is assumed to vary through the thickness of the plate in $x_{3}$-direction by power law function as below:

$$
\rho\left(x_{3}\right)=\rho_{m}+\left(\rho_{c}-\rho_{m}\right)\left(\frac{1}{2}-\frac{x_{3}}{h}\right)^{p}
$$

Since the dynamic pressure of the fluid is perpendicular to the plate surface, it appears only in $x_{3}$-direction as an applied load $P$ in Equation (8) and can be expressed as:

$$
P=m^{*} \frac{\partial^{2} U_{3}}{\partial t^{2}}=m^{*} \psi_{3}
$$


in which $m^{*}$, calculated in Section 2.2, is the added mass (AD) and dependent on fluid modelling and fluid-plate conditions.

Substituting the resultant forces and moments obtained from Equations (5) into Equation (8), the governing equations of motion for an FG rectangular plate are obtained as:

$$
\begin{aligned}
& \frac{D(1-v)}{2} \nabla^{2} \psi_{1}+\frac{D(1+v)}{2}\left(\psi_{1,1}+\psi_{2,2}\right)_{, 1}-\frac{A \kappa^{2}(1-v)}{2}\left(\psi_{1}-\psi_{3,1}\right) \\
& =I_{3} \dot{\psi} \\
& \frac{D(1-v)}{2} \nabla^{2} \psi_{2}+\frac{D(1+v)}{2}\left(\psi_{1,1}+\psi_{2,2}\right)_{, 2}-\frac{A \kappa^{2}(1-v)}{2}\left(\psi_{2}-\psi_{3,2}\right) \\
& =I_{3}^{2} \psi \\
& \quad \frac{A \kappa^{2}(1-v)}{2}\left[\nabla^{2} \psi_{3}-\left(\psi_{1,1}+\psi_{2,2}\right)\right]=\left(I_{1}+m^{*}\right)^{3} \psi
\end{aligned}
$$

For coding and derivational convenience, the following non-dimensional parameters are introduced:

$$
\begin{aligned}
& X_{1}=\frac{x_{1}}{a}, X_{2}=\frac{x_{2}}{b}, \eta=\frac{a}{b}, \delta=\frac{h}{a} \\
& \beta_{1}=\omega \sqrt{\frac{I_{3}}{A}}, \beta_{2}=a \omega \sqrt{\frac{I_{1}}{A}}, \delta^{2}=\frac{D}{A a^{2}} \\
& \gamma=\frac{m^{*}}{I_{1} h}
\end{aligned}
$$

in which $\beta_{1}$ and $\beta_{2}$ are named the eigenfrequency parameters. For a harmonic solution, the rotational and transverse displacements are assumed to be:

$$
\begin{aligned}
& \tilde{\psi}_{1}\left(X_{1}, X_{2}\right)=\psi_{1}\left(x_{1}, x_{2}, t\right) e^{-i \omega t} \\
& \tilde{\psi}_{2}\left(X_{1}, X_{2}\right)=\psi_{2}\left(x_{1}, x_{2}, t\right) e^{-i \omega t} \\
& \tilde{\psi}_{3}\left(X_{1}, X_{2}\right)=\frac{1}{a} \psi_{3}\left(x_{1}, x_{2}, t\right) e^{-i \omega t}
\end{aligned}
$$

where $\omega$ denotes the natural frequency of vibration in radians and $i=\sqrt{-1}$. Note that each parameter, having the over-bar, is dimensionless. Substituting Equations (13) and (14) into the governing Equation (12) yields:

$$
\begin{aligned}
& \tilde{\psi}_{1,11}+\eta^{2} \tilde{\psi}_{1,22}+\frac{v_{2}}{v_{1}}\left(\tilde{\psi}_{1,11}+\eta \tilde{\psi}_{2,12}\right)-\frac{A \kappa^{2} a^{2}}{D}\left(\tilde{\psi}_{1}-\tilde{\psi}_{3,1}\right)+\frac{I_{3} a^{2} \omega^{2}}{D v_{1}} \tilde{\psi}_{1}=0 \\
& \tilde{\psi}_{2,11}+\eta^{2} \tilde{\psi}_{2,22}+\frac{v_{2}}{v_{1}} \eta\left(\tilde{\psi}_{1,12}+\eta \tilde{\psi}_{2,22}\right)-\frac{A \kappa^{2} a^{2}}{D}\left(\tilde{\psi}_{2}-\eta \tilde{\psi}_{3,2}\right)+\frac{I_{3} a^{2} \omega^{2}}{D v_{1}} \tilde{\psi}_{2}=0 \\
& \tilde{\psi}_{3,11}+\eta^{2} \tilde{\psi}_{3,22}-\left(\tilde{\psi}_{1,1}+\eta \tilde{\psi}_{2,2}\right)-(1+\gamma) \frac{\beta_{2}^{2}}{\kappa^{2} v_{1}} \tilde{\psi}_{3}=0
\end{aligned}
$$


in which, $v_{1}=\frac{1-v}{2}$ and $v_{2}=\frac{1+v}{2}$.

The boundary conditions along the edges of the rectangular plate are defined in Table 1.

Equation (15) is the three coupled partial differential equations in terms of in-plane displacements, rotation functions and transverse displacements. For solving these equations analytically, it is desirable to find a method for decoupling them. Using the following analytical method, these governing equations will be decoupled. The general solution to Equation (14) in terms of the three dimensionless potential functions $W_{1}, W_{2}$ and $W_{3}$ may be expressed as (Hosseini-Hashemi et al., 2012):

$$
\begin{aligned}
& \tilde{\psi}_{1}=c_{1} W_{1,1}+c_{2} W_{2,1}-\eta W_{3,2} \\
& \tilde{\psi}_{2}=c_{1} \eta W_{1,2}+c_{2} \eta W_{2,2}+W_{3,1} \\
& \tilde{\psi}_{3}=W_{1}+W_{2}
\end{aligned}
$$

where:

$$
c_{1}=1-\frac{\alpha_{2}^{2}}{v_{1} \alpha_{3}^{2}} c_{2}=1-\frac{\alpha_{1}^{2}}{v_{1} \alpha_{3}^{2}}
$$

in which $\alpha_{1}^{2}, \alpha_{2}^{2}$ and $\alpha_{3}^{2}$ are the coefficients that may be determined using equations of motion and can be given after mathematical manipulation by:

$$
\begin{aligned}
& \alpha_{1}^{2}=\left(\frac{\beta_{1}^{2}}{\delta^{2}}-\frac{\beta_{2}^{2}(1+\gamma)}{k^{2} v_{1}}\right)+\sqrt{\left(\frac{\beta_{1}^{2}}{\delta^{2}}+\frac{\beta_{2}^{2}(1+\gamma)}{k^{2} v_{1}}\right)^{2}-\frac{\beta_{2}^{2}(1+\gamma)}{\delta^{2}}} \\
& \alpha_{2}^{2}=\left(\frac{\beta_{1}^{2}}{\delta^{2}}-\frac{\beta_{2}^{2}(1+\gamma)}{k^{2} v_{1}}\right)-\sqrt{\left(\frac{\beta_{1}^{2}}{\delta^{2}}+\frac{\beta_{2}^{2}(1+\gamma)}{k^{2} v_{1}}\right)^{2}-\frac{\beta_{2}^{2}(1+\gamma)}{\delta^{2}}} \\
& \alpha_{3}^{2}=-(1+\gamma)\left(\frac{k^{2}}{\delta^{2}}-\frac{\beta_{2}^{2}}{k^{2} v_{1}}\right)
\end{aligned}
$$

Equation (15) can be restated in terms of the three dimensionless potentials as follows:

$$
\begin{aligned}
& W_{1,11}+\eta^{2} W_{1,22}=-\alpha_{1}^{2} W_{1} \\
& W_{2,11}+\eta^{2} W_{2,22}=-\alpha_{2}^{2} W_{2} \\
& W_{3,11}+\eta^{2} W_{3,22}=-\alpha_{3}^{2} W_{3}
\end{aligned}
$$

One set of solutions to Equation (19) is taken as:

Table 1. Different boundary conditions of rectangular plate.

\begin{tabular}{lc}
\hline Edges & \multicolumn{1}{c}{$\begin{array}{c}\text { Edges } \\
X_{1}=0 \text { and } X_{1}=1\end{array}$} \\
\hline Simply supported $\bar{M}_{11}=\tilde{\psi}_{2}=\tilde{\psi}_{3}=0$ & Free edge: $\bar{M}_{22}=\bar{M}_{12}=\bar{Q}_{2}=0$ \\
& Simply supported: $\bar{M}_{22}=\tilde{\psi}_{1}=\tilde{\psi}_{3}=0$ \\
& Clamped edge: $\tilde{\psi}_{1}=\tilde{\psi}_{2}=\tilde{\psi}_{3}=0$ \\
\hline
\end{tabular}




$$
\begin{aligned}
W_{1}= & {\left[A_{1} \sin \left(\lambda_{1} X_{2}\right)+A_{2} \cos \left(\lambda_{1} X_{2}\right)\right] \sin \left(\mu_{1} X_{1}\right) } \\
& +\left[A_{3} \sin \left(\lambda_{1} X_{2}\right)+A_{4} \cos \left(\lambda_{1} X_{2}\right)\right] \sin \left(\mu_{1} X_{1}\right) \\
W_{2}= & {\left[A_{5} \sinh \left(\lambda_{2} X_{2}\right)+A_{6} \cosh \left(\lambda_{2} X_{2}\right)\right] \sin \left(\mu_{2} X_{1}\right) } \\
& +\left[A_{7} \sin \left(\lambda_{2} X_{2}\right)+A_{8} \cos \left(\lambda_{2} X_{2}\right)\right] \sin \left(\mu_{2} X_{1}\right) \\
W_{3}= & {\left[A_{9} \sinh \left(\lambda_{3} X_{2}\right)+A_{10} \cosh \left(\lambda_{3} X_{2}\right)\right] \cos \left(\mu_{3} X_{1}\right) } \\
& +\left[A_{11} \sin \left(\lambda_{3} X_{2}\right)+A_{12} \cos \left(\lambda_{3} X_{2}\right)\right] \cos \left(\mu_{3} X_{1}\right)
\end{aligned}
$$

In above equations, $\lambda_{j}$ and $\mu_{j}$ are related to $\alpha_{j}$ by following relationships:

$$
\begin{aligned}
& \alpha_{1}^{2}=\mu_{1}^{2}+\eta^{2} \lambda_{1}^{2} \\
& \alpha_{2}^{2}=\mu_{2}^{2}-\eta^{2} \lambda_{2}^{2} \\
& \alpha_{3}^{2}=\mu_{3}^{2}-\eta^{2} \lambda_{3}^{2}
\end{aligned}
$$

On the assumption of simply supported conditions at edges $X_{1}=0$ and $X_{1}=1$, Equation (20) is given by:

$$
\begin{aligned}
& W_{1}=\left[A_{1} \sin \left(\lambda_{1} X_{2}\right)+A_{2} \cos \left(\lambda_{1} X_{2}\right)\right] \sin \left(\mu_{1} X_{1}\right) \\
& W_{2}=\left[A_{5} \sinh \left(\lambda_{2} X_{2}\right)+A_{6} \cosh \left(\lambda_{2} X_{2}\right)\right] \sin \left(\mu_{2} X_{1}\right) \\
& W_{3}=\left[A_{9} \sinh \left(\lambda_{3} X_{2}\right)+A_{10} \cosh \left(\lambda_{3} X_{2}\right)\right] \cos \left(\mu_{3} X_{1}\right)
\end{aligned}
$$

in which $\mu_{1}=\mu_{2}=\mu_{3}=m \pi(m=1,2, \ldots)$

Introducing Equation (22) in Equation (16) and substituting the results into the appropriate boundary conditions along the edges $X_{2}=0$ and 1 lead to six homogenous equations. To obtain non-trivial solution of these equations, the determinant of coefficient matrix must be zero, which yields characteristic equations for rectangular Mindlin's FG plates with six combinations of boundary conditions, namely SSSS, SSSC, SCSC, SSSF, SFSF and SCSF.

\subsection{Formulation for fluid motion}

Generally, the fluid pressure acting upon the structure is expressed as a function of acceleration. The fluid force matrices are superimposed onto the structural matrices to form the dynamic equations of a coupled fluidstructure system. Linear potential flow is applied to describe the fluid effect that leads to the fluid dynamic forces. The mathematical model is based on the following assumptions: (i) the fluid flow is potential; (ii) vibration is linear; (iii) since the flow is inviscid, there is no shear, and the fluid pressure is purely normal to the plate wall; and (v) the fluid is incompressible. Based on the aforementioned hypothesis, the potential function, which satisfies the Laplace equation, is expressed in the Cartesian coordinate system as (Myung \& Young, 2003): 


$$
\nabla^{2} \Phi=\frac{\partial^{2} \Phi}{\partial x_{1}^{2}}+\frac{\partial^{2} \Phi}{\partial x_{2}^{2}}+\frac{\partial^{2} \Phi}{\partial x_{3}^{2}}=0
$$

where $\Phi$ is the potential flow function. Using Bernoulli's equation, the fluid pressure at the solid-fluid interface may be expressed as:

$$
p_{L}=\left.p\right|_{x_{3}=\frac{-h}{2}}=-\left.\rho_{f} \frac{\partial}{\partial t}\right|_{x_{3}=\frac{-h}{2}}
$$

where $\rho_{f}$ is the fluid density. The dynamic displacement of the fluid normal to the plate must coincide with that of the plate to satisfy the continuity requirement. This condition implies a permanent contact between the plate surface and the peripheral fluid layer. So, compatibility condition between plate and the fluid becomes:

$$
\left(\frac{\partial \phi}{\partial x_{3}}\right)_{x_{3}=-\frac{h}{2}}=\frac{\partial U_{3}}{\partial t}
$$

The following separate variable relation is assumed for the potential velocity function:

$$
\phi\left(x_{1}, x_{2}, x_{3}, t\right)=F\left(x_{3}\right) G\left(x_{1}, x_{2}, t\right)
$$

where $F\left(x_{3}\right)$ and $G\left(x_{1}, x_{2}, t\right)$ are two separate functions to be determined.

Using Equations (24) and (25) and introducing Equation (26) into Equation (25), the potential velocity on the bottom surface of the plate is obtained as follows:

$$
\Phi\left(x_{1}, x_{2}, x_{3},\right)=\frac{F\left(x_{3}\right)}{\left.\frac{d F}{d x_{3}}\right|_{x_{3}=\frac{-h}{2}}} \frac{\partial U_{3}}{\partial t}
$$

Substituting Equation (27) into relation (23) leads to the following differential equation of second order:

$$
\frac{d^{2} F\left(x_{3}\right)}{d x_{3}{ }^{2}}-\mu_{f}^{2} F\left(x_{3}\right)=0
$$

in which $\mu_{f}$ is a plane wave number, which is determined by the vibrating frequency of the plate in contact with fluid and fluid boundary conditions in the $x_{1}$ and $x_{2}$ directions as $\mu_{f}=\sqrt{\mu_{1}^{2}+\mu_{2}^{2}}$ (Myung \& Young, 2003). Kerboua et al. (2008) used a simple form of $\mu_{f}=(\pi / a) \sqrt{1+\eta^{2}}$ for all boundary conditions. Though the value of $\mu_{f}$ varies for various boundary conditions, they ignored the effect of boundary conditions on the $\mu_{f}$. In addition, the wave number presented in Kerboua et al. (2008) is independent of air frequency and is constant when mode number changes. In this study, the wave number parameter proposed in Haddara and Cao (1996) is modified using Mindlin's 
plate parameters. It is worthwhile to mention that when higher degrees of edge constraint are applied to the other two edges of the rectangular plate (in the order from free to simply supported to clamped), the variation of the $\mu \mathrm{f}$ is more tangible (e.g., from SFSF to SCSC).

The following wave number parameters are introduced when at least one of the rectangular plate edges can freely vibrate (i.e. SFSF, SCSF and SSSF):

$$
\mu_{1}=\sqrt{\alpha^{2}-\left(\frac{n \pi}{a}\right)^{2}}, \mu_{2}=\frac{2 \pi}{L}
$$

where:

$$
\alpha^{2}=\frac{\beta_{a}^{2}}{2 a^{2}}\left[\left(\frac{\beta_{1}^{2}}{\delta^{2}}-\frac{\beta_{2}^{2}}{k^{2} v_{1}}\right)+\sqrt{\left(\frac{\beta_{1}^{2}}{\delta^{2}}+\frac{\beta_{2}^{2}}{k^{2} v_{1}}\right)^{2}-\frac{\beta_{2}^{2}}{\delta^{2}}}\right]
$$

In the above equations, $L$ is width of tank and $\beta_{a}$ is dimensionless frequency parameter in vacuum. For other three boundary conditions (i.e. SSSS, SCSC and SSSC), $\mu_{2}$ in Equation (29) is replaced with $\mu_{2}=m \pi / a$, while $\mu_{1}$ will remain unchanged. Note that when $\mu_{1}$ takes a complex value, $\bar{\mu}_{1}$ must be used instead of that as $\bar{\mu}_{1}=\sqrt{-\alpha^{2}+\left(\frac{n \pi}{a}\right)^{2}}$.

The general solution of Equation (28) is given as:

$$
F\left(x_{3}\right)=B_{1} e^{\mu_{f} x_{3}}+B_{2} e^{-\mu_{f} x_{3}}
$$

where $B_{1}$ and $B_{2}$ are two unknown constants. Substituting Equation (29) into Equation (27), one gets the following expression for the potential function:

$$
\Phi\left(x_{1}, x_{2}, x_{3},\right)=\frac{B_{1} e^{\mu_{f} x_{3}}+B_{2} e^{-\mu_{f} x_{3}}}{\left.\frac{d F}{d x_{3}}\right|_{x_{3}=\frac{-h}{2}}} \frac{\partial U_{3}}{\partial t}
$$

The boundary conditions on the rigid bottom assure the zero velocity:

$$
\left.\frac{\partial}{\partial x_{3}}\right|_{x_{3}=-h_{1}-\frac{h}{2}}=0
$$

Introducing Equation (32) into Equation (33), the potential function is obtained as follows:

$$
\Phi=\frac{e^{\mu_{f} x_{3}}+c_{1} e^{-\mu_{f} x_{3}}}{\mu_{f}\left(e^{-\mu_{f} \frac{h}{2}}-c_{1} e^{\mu_{f} \frac{h}{2}}\right.} \frac{\partial U_{3}}{\partial t}
$$


where:

$$
c_{1}=e^{-2 \mu_{f}\left(h_{1}+\frac{h}{2}\right)}
$$

By inserting Equation (34) into Equation (24), the pressure acting upon the bottom surface of the plate can be expressed as:

$$
p_{L}=-\frac{\rho_{f}}{\mu_{f}}\left[\frac{1+c_{1} e^{\mu_{f} h}}{1-c_{1} e^{\mu_{f} h}}\right] \frac{\partial^{2} U_{3}}{\partial t^{2}}=m^{*} \frac{\partial^{2} U_{3}}{\partial t^{2}}
$$

\section{Results and discussion}

In order to validate the present method, first the numerical results of nondimensional frequency parameter $\bar{\omega}=\omega\left(b^{2} / h\right) \sqrt{\rho / E}$ are compared with the results of Hosseini-Hashemi et al. (2012) for homogeneous and isotropic rectangular plate in Table 1 with SSSS boundary condition and square plate coupled with cubic volume of fluid $(\delta=0.05$ and $\eta=1)$. Also, the finite element model of FG rectangular plate coupled with fluid has been analysed with $\eta=2, \delta=0.05, h_{1} / a=0.4$ and $v=0.3$. The nondimensional frequency parameter $\beta=\omega a \sqrt{\rho_{c} / E_{c}}$ for the FG rectangular plates with various boundary conditions is compared with results of FEM in Table 3. It can be found from Tables 2 and 3 that the present results are in good agreement with those of Hosseini-Hashemi et al. (2012) and with the FEM results. It is necessary to mention that values of wave number $\mu_{f}$ have a remarkable effect on added mass $m^{*}$ in Equation (36). This parameter is constant and it is depend on problem conditions. It seems that the differences between results in Table 2 are related to choosing of this parameter.

Table 2. Comparison of the first six frequency parameters $\bar{\omega}=\omega\left(b^{2} / h\right) \sqrt{\rho / E}$ for a homogeneous square plate coupled with cubic volume of fluid with Hosseini-Hashemi et al. (2012).

\begin{tabular}{llcccccc}
\hline B.C. & \multicolumn{1}{c}{ Method } & $\beta_{1}$ & $\beta_{2}$ & $\beta_{3}$ & $\beta_{4}$ & $\beta_{5}$ & $\beta_{6}$ \\
\hline SSSS & Hosseini-Hashemi et al. (2012) & 11.620 & 38.681 & 38.681 & 65.477 & 75.877 & 78.502 \\
& Present & 11.980 & 40.106 & 40.106 & 67.414 & 80.102 & 83.254 \\
\hline
\end{tabular}

Table 3. Comparison of the first six frequency parameters $\beta=\omega a \sqrt{\rho_{c} / E_{c}}$ for an FG square plate coupled with cubic volume of fluid with FE model.

\begin{tabular}{llllllll}
\hline B.C. & Method & $\beta_{1}$ & $\beta_{2}$ & $\beta_{3}$ & $\beta_{4}$ & $\beta_{5}$ & \multicolumn{1}{c}{$\beta_{6}$} \\
\hline SSSS & Present & 1.13 & 1.80 & 2.91 & 3.79 & 4.38 & 5.80 \\
& FEM & 1.16 & 1.84 & 3.37 & 4.26 & 4.46 & 6.50 \\
SCSC & Present & 2.61 & 6.13 & 4.91 & 8.30 & 12.91 & 18.93 \\
& FEM & 2.66 & 6.23 & 5.44 & 9.20 & 14.32 & 20.05 \\
SFSF & Present & 1.06 & 1.76 & 3.99 & 4.24 & 4.95 & 7.54 \\
& FEM & 1.08 & 1.81 & 4.34 & 4.76 & 5.84 & 8.50 \\
\hline
\end{tabular}


For numerical results, an FGM composed of aluminium (as metal) and alumina (as ceramic) is considered. The mechanical properties of plate are: Young's modulus and density of aluminium $E_{m}=70 \mathrm{GPa}, \rho_{m}=$ $2700 \mathrm{~kg} / \mathrm{m}^{3}$ and Young's modulus and density of ceramic are ceramic $E_{c}=380 \mathrm{GPa}, \rho_{c}=4500 \mathrm{~kg} / \mathrm{m}^{3}$. The Poisson's ratio of the plate is assumed to be constant through the thickness and equal to 0.3 , and the density of the fluid is assumed to be $1000 \mathrm{~kg} / \mathrm{m}^{3}$.

The first four non-dimensional frequencies $\beta=\omega a \sqrt{\rho_{c} / E_{c}}$ are shown for three boundary conditions SSSS, SCSC and SFSF, respectively, in Tables 4-6 for different power law index $(p)$ and thickness/length ratio $h / a$. Other parameters are assumed to be $\eta=1, h_{1} / a=0.4$ and $v=0.3$.

It can be seen that for all boundary conditions, the natural frequencies decrease by increasing the ratio thickness to length $h / a$. Also, by increasing the power law index $p$, the natural frequencies decrease.

In Figure 2, the variation of the non-dimensional frequency parameter $\beta$ against the thickness to length ratio $\delta$ for the various boundary conditions of the plate is plotted. It is seen from Figure 2 that with the increase of the thickness to length ratio $\delta$, the non-dimensional frequency parameter $\beta$ generally decreases for any boundary conditions of the plate. Moreover, decreasing of $\beta$ for boundary condition SCSC is greater than other two boundary conditions.

The variation of the non-dimensional frequency parameter $\beta$ versus the thickness to length ratio $\delta$ is presented for some values of $h_{1} / a, p=2$ and $\eta=2$ (Fig. 3). It can obviously be seen that the non-dimensional frequency parameter $\beta$ initially increases when the thickness to length ratio $\delta$ varies between 0.05 and 0.1 and then decreases when $\delta>0.1$.

The effect of the length to width ratio of the plate $\eta$ on the nondimensional frequency parameter $\beta$ is shown in Figure 4 for different values of power law index $p, h_{1} / a=0.4$ and $\delta=0.02$. It can be observed

Table 4. First four frequency parameters $\beta$ of SSSS FG square plate coupled with cubic volume of fluid for different values of $h / a$ and $p$.

\begin{tabular}{lcccccc}
\hline & \multirow{5}{*}{$\begin{array}{c}\text { Frequency } \\
\text { pramet }\end{array}$} & 0 & 0.5 & 1 & 2 & 5 \\
\cline { 3 - 6 } & paramer & 3.4409 & 2.9322 & 2.6473 & 2.4017 & 2.2528 \\
& $\beta_{1}$ & 5.2802 & 4.5122 & 4.0773 & 3.6953 & 3.4492 \\
& $\beta_{2}$ & 8.0710 & 6.9231 & 6.2636 & 5.6695 & 5.2579 \\
& $\beta_{3}$ & 9.7416 & 8.6926 & 7.8711 & 7.1189 & 6.5749 \\
0.1 & $\beta_{4}$ & 3.6518 & 3.0983 & 2.7937 & 2.5386 & 2.3998 \\
& $\beta_{1}$ & 5.7693 & 4.8997 & 4.4192 & 4.0142 & 3.7881 \\
& $\beta_{2}$ & 9.1876 & 7.8145 & 7.0512 & 6.4015 & 6.0247 \\
& $\beta_{3}$ & 11.831 & 10.074 & 9.0928 & 8.2515 & 7.7505 \\
0.2 & $\beta_{4}$ & 3.7123 & 3.1456 & 2.8352 & 2.5777 & 2.4425 \\
& $\beta_{1}$ & 5.9198 & 5.0175 & 4.5228 & 4.115 & 3.8939 \\
& $\beta_{2}$ & 9.5668 & 8.1121 & 7.3132 & 6.6471 & 6.2903 \\
& $\beta_{3}$ & 12.456 & 10.566 & 9.5261 & 8.6572 & 8.1875 \\
\hline
\end{tabular}


Table 5. First four frequency parameters $\beta=\omega a^{2} \sqrt{\rho_{c} / E_{c}} / h$ of SCSC FG square plate coupled with cubic volume of fluid for different values of $h / a$ and $p$.

\begin{tabular}{ccccccc}
\hline & \multirow{5}{*}{$\begin{array}{c}\text { Frequency } \\
\text { parameter }\end{array}$} & 0 & 0.5 & 1 & 2 & 5 \\
\cline { 3 - 6 } 0 & $\beta_{1}$ & 6.7663 & 5.8409 & 5.3039 & 4.8032 & 4.4127 \\
& $\beta_{2}$ & 12.060 & 10.420 & 9.4561 & 8.5466 & 7.8331 \\
& $\beta_{3}$ & 13.501 & 11.758 & 10.712 & 9.6759 & 8.7548 \\
& $\beta_{4}$ & 17.718 & 15.423 & 14.040 & 12.670 & 11.473 \\
0.1 & $\beta_{1}$ & 8.0702 & 6.8847 & 6.2222 & 5.6494 & 5.2930 \\
& $\beta_{2}$ & 14.862 & 12.693 & 11.472 & 10.405 & 9.7255 \\
& $\beta_{3}$ & 17.918 & 15.371 & 13.921 & 12.621 & 11.714 \\
& $\beta_{4}$ & 23.850 & 20.475 & 18.543 & 16.799 & 15.566 \\
0.2 & $\beta_{1}$ & 8.5674 & 7.2715 & 6.5585 & 5.9612 & 5.6332 \\
& $\beta_{2}$ & 16.065 & 13.642 & 15.390 & 11.181 & 10.555 \\
& $\beta_{3}$ & 20.050 & 17.051 & 12.305 & 13.981 & 13.167 \\
& $\beta_{4}$ & 27.100 & 23.060 & 20.816 & 18.904 & 17.785 \\
\hline
\end{tabular}

Table 6. First four frequency parameters $\beta$ of SFSF FG square plate coupled with cubic volume of fluid for different values of $h / a$ and $p$.

\begin{tabular}{ccccccc}
\hline & & \multicolumn{5}{c}{ Power law index $(p)$} \\
\cline { 3 - 6 }$h / a$ & $\begin{array}{c}\text { Frequency } \\
\text { parameters }\end{array}$ & 0 & 0.5 & 1 & 2 & 5 \\
\hline 0.05 & $\beta_{1}$ & 2.7184 & 2.5672 & 2.5096 & 2.5006 & 2.4429 \\
& $\beta_{2}$ & 4.2650 & 4.0376 & 3.9474 & 3.9260 & 3.8764 \\
& $\beta_{3}$ & 8.8171 & 8.3769 & 8.1902 & 8.1217 & 8.0770 \\
& $\beta_{4}$ & 9.4629 & 8.9983 & 8.8020 & 8.7282 & 8.6752 \\
0.1 & $\beta_{1}$ & 2.8569 & 2.6905 & 2.6296 & 2.6253 & 2.5820 \\
& $\beta_{2}$ & 4.6570 & 4.3908 & 4.2915 & 4.2811 & 4.1656 \\
& $\beta_{3}$ & 10.246 & 9.6764 & 9.4582 & 9.4233 & 9.3830 \\
& $\beta_{4}$ & 11.002 & 10.390 & 10.157 & 10.121 & 10.092 \\
0.2 & $\beta_{1}$ & 2.8977 & 2.7268 & 2.6648 & 2.6620 & 2.5233 \\
& $\beta_{2}$ & 4.7986 & 4.5176 & 4.4150 & 4.4090 & 4.3071 \\
& $\beta_{3}$ & 10.809 & 10.182 & 9.9505 & 9.9330 & 9.145 \\
& $\beta_{4}$ & 11.561 & 10.888 & 10.641 & 10.624 & 10.553 \\
\hline
\end{tabular}

that the non-dimensional frequency parameter $\beta$ decreases by increasing $\eta$ for all values of $p$.

The influence of the aspect ratio length to width of the plate $\eta$ on the non-dimensional frequency parameter $\beta$ for constant value of thickness/ length ratio $(\delta=0.02)$ and power law index $(p=2)$ of rectangular plate coupled with cubic fluid is presented in Figure 5. It is observed that by increasing the aspect ratio $\eta$, the non-dimensional frequency parameter increases for boundary conditions SCSC and SSSS and decreases for boundary condition SFSF. It can also be inferred from Figure 5 that increasing of the non-dimensional frequency parameter for boundary condition SCSC is greater than that for SSSS.

In order to study the effects of the fluid density on the vibrational behaviour of the rectangular FG plate coupled with fluid, the non-dimensional frequencies are listed for boundary condition SFSF in Table 7 with $p=2, h_{1} / a=0.4$ and $\eta=2$. As expected, it can be found that by increasing the fluid density, the non-dimensional frequency parameter $\beta$ decreases. 


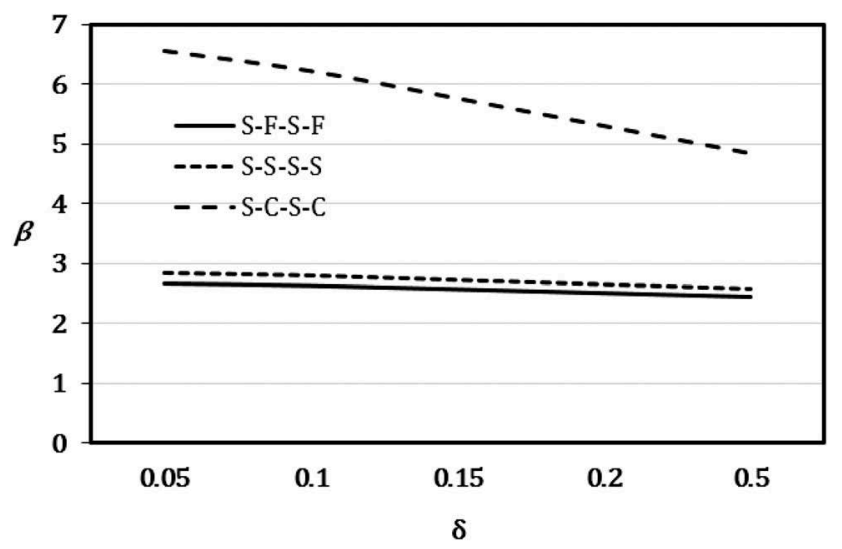

Figure 2. The non-dimensional frequency parameter $\beta$ versus the thickness to length ratio $\delta$ for the three boundary conditions of the FG rectangular plate when $p=2, h_{1} / a=0.4$ and $\eta=2$.

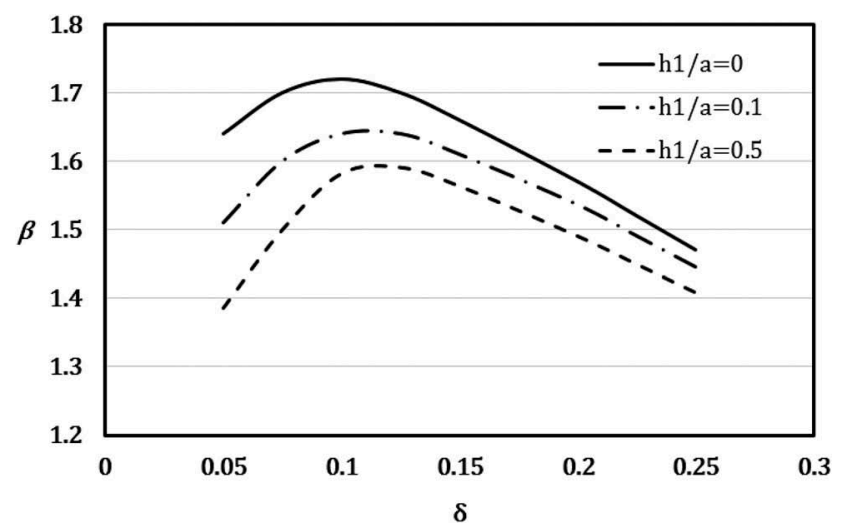

Figure 3. The non-dimensional frequency parameter $\beta$ versus the thickness to length ratio $\delta$ for SCSF FG rectangular plate when $p=2$ and $\eta=2$.

\section{Conclusions}

The aim of this paper is to present an analytical solution for the free vibration analysis of moderately thick FG rectangular plate coupled with incompressible fluid. The governing equations of motion have been derived based on the first-order shear deformation plate theory, and the equation governing on the oscillatory behaviour of fluid has been obtained by solving Laplace equation with satisfying boundary conditions. These equations are five highly coupled partial differential equations that have been decoupled by some mathematical manipulations. The decoupled equations have been solved analytically for the FG rectangular plate with various boundary conditions in edges. By solving the governing equations on fluid-plate interactions and satisfying the boundary conditions in edges, the natural 


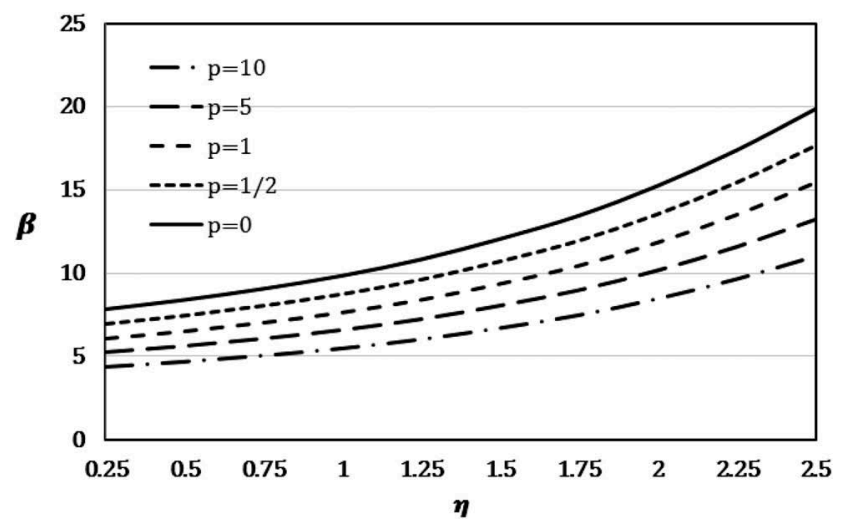

Figure 4. The non-dimensional frequency parameter $\beta$ versus the length to width ratio $\eta$ for SCSF FG rectangular plate when $h_{1} / a=0.4$ and $\delta=0.02$.

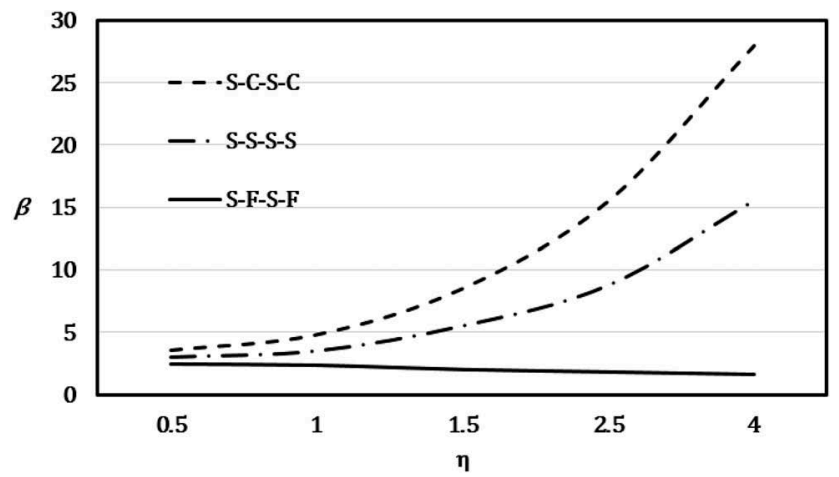

Figure 5. The non-dimensional frequency parameter $\beta$ versus the length to width ratio $\eta$ for the three boundary conditions of the FG rectangular plate when $p=1, h_{1} / a=0.4$ and $\delta=0.005$.

Table 7. First five frequency parameters $\beta=\omega a^{2} \sqrt{\rho_{c} / E_{c}} / h$ of SFSF FG square plate coupled with cubic volume of fluid for different values $\rho_{f}$.

\begin{tabular}{lcccccc}
\hline Fluid & $\rho_{f} \frac{\mathrm{kg}}{\left(\mathrm{m}^{3}\right)}$ & $\beta_{1}$ & $\beta_{2}$ & $\beta_{3}$ & $\beta_{4}$ & $\beta_{5}$ \\
\hline Gasoline $\left(16^{\circ} \mathrm{C}\right)$ & 737.22 & 3.2672 & 5.8625 & 12.576 & 14.235 & 16.189 \\
Kerosene $\left(16^{\circ} \mathrm{C}\right)$ & 817.15 & 2.8535 & 4.5726 & 10.221 & 12.321 & 14.852 \\
Water pure $\left(4^{\circ} \mathrm{C}\right)$ & 1000 & 2.6512 & 4.3958 & 9.9213 & 10.602 & 12.153 \\
Sea water $\left(25^{\circ} \mathrm{C}\right)$ & 1025 & 2.6253 & 2.8628 & 8.9592 & 9.9125 & 11.425 \\
\hline
\end{tabular}

frequencies of plate have been obtained in various cases. The natural frequencies of the rectangular plate for different boundary conditions with several aspect ratios, different thickness/length ratios and some power law indices have been tabulated. The following conclusions can be remarked: 
(1) As the power law index increases, the natural frequencies of the FG rectangular plate coupled with fluid decrease for all ratios of thickness/length and boundary conditions.

(2) By increasing the length to width ratio of the rectangular plate, the non-dimensional frequency parameter increases for SCSC and SSSS boundary conditions.

(3) By increasing the length to width ratio of the rectangular plate, the non-dimensional frequency parameter decreases for SFSF boundary condition.

(4) The value of the natural frequency initially increases when the thickness to length ratio $\delta$ varies between 0.05 and 0.1 and then decreases when $\delta>0.1$ for boundary conditions SSSS, SFSF and SCSC.

(5) The non-dimensional frequency parameter decreases by increasing the length to width ratio of the plate for boundary condition SFSF and all values of power law indexes.

(6) By increasing the fluid density, the non-dimensional frequency parameter decreases.

\section{Disclosure statement}

No potential conflict of interest was reported by the authors.

\section{References}

Allahverdizadeh, A., Naei, M. H., \& Bahrami, M. N. (2008). Nonlinear free and forced vibration analysis of thin circular functionally graded plates. Journal of Sound and Vibration, 310(4), 966-984.

Amabili, M., Frosali, G., \& Kwak, M. K. (1996). Free vibrations of annular plates coupled with fluids. Journal of Sound and Vibration, 191(5), 825-846.

Dong, C. Y. (2008). Three-dimensional free vibration analysis of functionally graded annular plates using the Chebyshev-Ritz method. Materials \& Design, 29(8), 1518-1525.

Haddara, M. R., \& Cao, S. (1996). A study of the dynamic response of submerged rectangular flat plates. Marine Structures, 9(10), 913-933.

Hashemi, S. H., Karimi, M., \& Taher, H. R. D. (2010). Vibration analysis of rectangular Mindlin plates on elastic foundations and vertically in contact with stationary fluid by the Ritz method. Ocean Engineering, 37(2), 174-185.

Hasheminejad, S. M., Khaani, H. A., \& Shakeri, R. (2013). Free vibration and dynamic response of a fluid-coupled double elliptical plate system using Mathieu functions. International Journal of Mechanical Sciences, 75, 66-79.

Hejripour, F., \& Saidi, A. R. (2012). Nonlinear free vibration analysis of annular sector plates using differential quadrature method. Proceedings of the Institution of Mechanical Engineers, Part C: Journal of Mechanical Engineering Science, 226(2), 485-497. 
Hosseini-Hashemi, S., Karimi, M., \& Rokni, H. (2012). Natural frequencies of rectangular Mindlin plates coupled with stationary fluid. Applied Mathematical Modelling, 36(2), 764-778.

Hosseini-Hashemi, S., Taher, H. R. D., Akhavan, H., \& Omidi, M. (2010). Free vibration of functionally graded rectangular plates using first-order shear deformation plate theory. Applied Mathematical Modelling, 34(5), 1276-1291.

Jeong, K. H. (2003). Free vibration of two identical circular plates coupled with bounded fluid. Journal of Sound and Vibration, 260(4), 653-670.

Jeong, K. H., Lee, G. M., \& Kim, T. W. (2009). Free vibration analysis of a circular plate partially in contact with a liquid. Journal of Sound and Vibration, 324(1), 194-208.

Jomehzadeh, E., Saidi, A. R., \& Atashipour, S. R. (2009). An analytical approach for stress analysis of functionally graded annular sector plates. Materials \& Design, 30(9), 36793685.

Kerboua, Y., Lakis, A. A., Thomas, M., \& Marcouiller, L. (2008). Vibration analysis of rectangular plates coupled with fluid. Applied Mathematical Modelling, 32(12), 25702586.

Khorshidi, K., \& Bakhsheshy, A. (2014). Free natural frequency analysis of an FG composite rectangular plate coupled with fluid using Rayleigh-Ritz method. Mechanics of Advanced Composite Structures, 1(2), 131-143.

Kwak, M. K. (1997). Hydroelastic vibration of circular plates. Journal of Sound and Vibration, 201(3), 293-303.

Myung, J. J., \& Young, H. C. (2003). Fluid bounding effect on natural frequencies of fluidcoupled circular plates. KSME International Journal, 17(9), 1297-1315.

Tariverdilo, S., Shahmardani, M., Mirzapour, J., \& Shabani, R. (2013). Asymmetric free vibration of circular plate in contact with incompressible fluid. Applied Mathematical Modelling, 37(1), 228-239.

Uğurlu, B., Kutlu, A., Ergin, A., \& Omurtag, M. H. (2008). Dynamics of a rectangular plate resting on an elastic foundation and partially in contact with a quiescent fluid. Journal of Sound and Vibration, 317(1), 308-328. 